\title{
The secretory senescence of the oro-pharyngo-laryngeal tract
}

Andrea Gallo ${ }^{1}$, Veronica Clemenzi ${ }^{1}$, Andrea Stolfa ${ }^{1}$, Giulio Pagliuca², Ferdinando Maria Nobili Benedetti ${ }^{2}$, Claudio Caporale ${ }^{3}$, Alessandro Maselli del Giudice ${ }^{4}$, Teresa Maino ${ }^{5}$, Valentina de Robertis ${ }^{5}$, Francesco Cariti ${ }^{4}$, Francesco Barbara ${ }^{4}$

${ }^{1}$ Department of Sensorial Organs, ENT Section, Sapienza University of Rome, Italy; ${ }^{2}$ ENT University Unit, ASL Latina, Italy; ${ }^{3}$ ENT Unit of Santo Spirito Hospital, Pescara, Italy; ${ }^{4}$ Department of Otorhinolaryngology "Mons. Dimiccoli", Barletta, Italy; ${ }^{5}$ ENT Unit, Policlinico di Bari, Italy

Saliva is an essential body fluid. It is important in maintaining oral health, taste acuity, mastication, deglutition, digestion, regulation of oral flora, oral cleansing, voice acuity, and speech articulation. In the elderly, the glandular parenchyma undergoes involution of the cellular component, and this leads to a reduction in the production of saliva. Qualitative and quantitative alterations of this biological fluid can cause patient discomfort and quality of life decline. This often translates into xerostomia, whose real meaning is mouth dryness. We will discuss here causes of xerostomia in the elderly, such as drugs, head and neck radiotherapy, and autoimmune diseases.

Received: April 14, 2020

Accepted: June 10, 2020

\section{Correspondence}

Andrea Gallo

Department of Sensorial Organs, ENT Section, Sapienza University of Rome, piazzale A. Moro 5, 00185, Rome, Italy. E-mail: andrea.gallo@ uniroma1.it

\section{Conflict of interest}

The Authors declare no conflict of interest

How to cite this article: Gallo A, Clemenzi $V$, Stolfa $A$, et al. The secretory senescence of the oro-pharyngo-laryngeal tract. Journal of Gerontology and Geriatrics 2020;68:69-76. https://doi. org/10.36150/2499-6564-483

C Copyright by Società Italiana di Gerontologia e Geriatria (SIGG)

\section{(c) (1) $(9)$}

\section{OPEN ACCESS}

This is an open access article distributed in accordance with the CC-BY-NC-ND (Creative Commons Attribution-NonCommercial-NoDerivatives 4.0 International) license. The article can be used by giving appropriate credit and mentioning the license, but only for non-commercial purposes and only in the original version. For further information: https://creativecommons.org/licenses/by-nc-nd/4.0/deed.en
Key words: salivary glands, oral cavity, xerostomia, senescence, secretory system, pharyngo-laryngeal tract, immune defence

\section{THE ORAL CAVITY AND SALIVARY GLANDS}

\section{SALIVARY GLAND PHYSIOLOGY}

Salivary secretion is a hypotonic solution composed mainly of water, electrolytes $\left(\mathrm{Na}^{+}, \mathrm{K}^{+}, \mathrm{Ca}^{2+}, \mathrm{Mg}^{2+}, \mathrm{HCO}_{3}{ }^{-}\right)$and organic compounds such as immunoglobulins (IgG, $\operatorname{lgM}$, IgA), proteins, enzymes, mucins, urea, and ammonium. Its $\mathrm{pH}$ is between 6 and 7 . Ninety percent of saliva is produced by the major salivary glands, while the remaining $10 \%$ is secreted by the minor salivary glands situated in the lining of the upper aerodigestive tract. Various systemic diseases, many drugs and oncological therapies can influence the amount of salivary secretion. All these factors can significantly compromise oral health ${ }^{1}$.

Physiological basal saliva production in adults ranges from 0.3 to $0.5 \mathrm{ml} /$ min. Secretion upon stimulation can reach values of up to $2 \mathrm{ml} / \mathrm{min}$. Hyposalivation can be defined when basal salivary secretion values are lower than $0.1 \mathrm{ml} / \mathrm{min}$ and $0.7 \mathrm{ml} / \mathrm{min}$ upon stimulus.

Both afferent and efferent stimuli modulate neural control of salivation. Taste and mastication play a key role in saliva production, however, smell, sight, and the thought of food also contribute. Input to the solitary nucleus from afferent stimuli is integrated via the facial (VII) and glossopharyngeal (IX) nerves. Parasympathetic efferent pathways for the sublingual and submandibular glands are from the facial nerve via the submandibular ganglion and from the glossopharyngeal nerve via 
the otic ganglion for the parotid gland. Through the liberation of acetylcholine, the parasympathetic nervous system acts upon the muscarinic M3 receptors and produces abundant aqueous saliva. Sympathetic post-ganglionic pathways originate from the cervical ganglion of the sympathetic chain. During stimulation of the sympathetic nervous system, norepinephrine binds the $\beta$-adrenergic receptors and causes the production of a thicker and less abundant secretion ${ }^{2-4}$. A two-step process characterizes saliva production and secretion ${ }^{4,5}$. First, secretion of the isotonic plasmalike primary saliva fluid takes place in acinar cells. A vectorial ion transport develops through ion channels and transporters from the serosal (basolateral) to the luminal (apical) side in the secretory direction. The $\mathrm{Cl}^{-}$transporting proteins expressed on the basolateral membrane accumulate $\mathrm{Cl}^{-}$in amounts greater than its equilibrium potential. Water movement in the salivary glands can follow the transcellular secretion of $\mathrm{Cl}^{-}$. During the second phase, the passage along the duct system modifies the $\mathrm{NaCl}$-rich fluid; actually, most of the $\mathrm{NaCl}$ is reabsorbed. The $\mathrm{K}^{+}$concentration in saliva is higher than in plasma and it is due to $\mathrm{KHCO}_{3}$ secretion. The ductal epithelium is almost waterproof, so the final saliva is usually hypotonic; moreover, $\mathrm{NaCl}$ reabsorption is greater than $\mathrm{KHCO}_{3}$ secretion. This biochemical process forms the final saliva, which is composed of water (99\%), electrolytes and organic components.

Qualitative and quantitative alterations of this biological fluid can cause patient discomfort and quality of life decline. It is therefore necessary to introduce the concept of xerostomia, meaning mouth dryness.

\section{EPIDEMIOLOGY OF SALIVARY GLAND DYSFUNCTION}

It is quite difficult to define the worldwide estimate of xerostomia and salivary gland dysfunction due to methodological differences in study populations and diagnostic criteria. Certainly, this condition arises more frequently in females than in males, and in the elderly compared to young people. The prevalence is probably approximately $30 \%$ of the population aged 65 and older. The most frequent cause of xerostomia are drugs, followed by Sjogren's Syndrome (SS) and head and neck radiotherapy (RT). However, it is not easy to find the true prevalence of xerostomia in the elderly who are under medication. In patients with SS, an autoimmune disease that affects 1-4\% of older adult populations, the prevalence of xerostomia is nearly $100 \%$.

Treatment of head and neck cancer causes permanent xerostomia with a $100 \%$ prevalence rate. In the United States, approximately 30,000 cases of head and neck cancers were diagnosed in 2000 , and the majority of these patients underwent RT causing them permanent salivary gland dysfunction and xerostomia. Estimates of the prevalence of xerostomia in nursing homes and in adult ambulatory populations range $16-72 \%{ }^{6}$.

\section{Clinical findings of Salivary dYSFUnction}

As for the physiopathological process, it is possible to say that in the elderly the glandular parenchyma undergoes involution of the cellular component leading to a reduction in the production of saliva. Studies performed on salivary gland specimens have shown that senescence leads to a $30-50 \%$ reduction in the number of acinar cells and to the replacement of the parenchyma with adipose tissue. This glandular degeneration begins as early as 35 years of age and continues until 75 . The glandular repair process in elderly patients is slower than in young subjects, and this is confirmed by the frequent finding of xerostomia in geriatric patients with a history of radiation therapy ${ }^{7}$.

Moreover, elderly people frequently suffer from multiple pathologies which often require polypharmacological therapy. Xerostomia predisposes patients to an alteration of the oral microbial flora with the increase in virulent bacterial and fungal activity. Loss of salivary gland function usually results in the appearance of an increased number of caries forming bacteria (mutans Streptococci and Lactobacillus) in the oral cavity, resulting in "radiation caries" caused by the loss of buffering capacity, lowered salivary $\mathrm{pH}$ levels, loss of mechanical flushing, and decreased production of salivary immunoglobulins (i.e., IgA, lgG, IgM), lysozymes, and peroxidases (Fig. 1). Xerostomia may result in mucositis, oral pain or discomfort, and difficulty with articulation, chewing and deglutition; it is also associated with increased dysgeusia, ageusia, soft tissue breakdown, bone loss, and chronic infection. Often patients complain of an oral

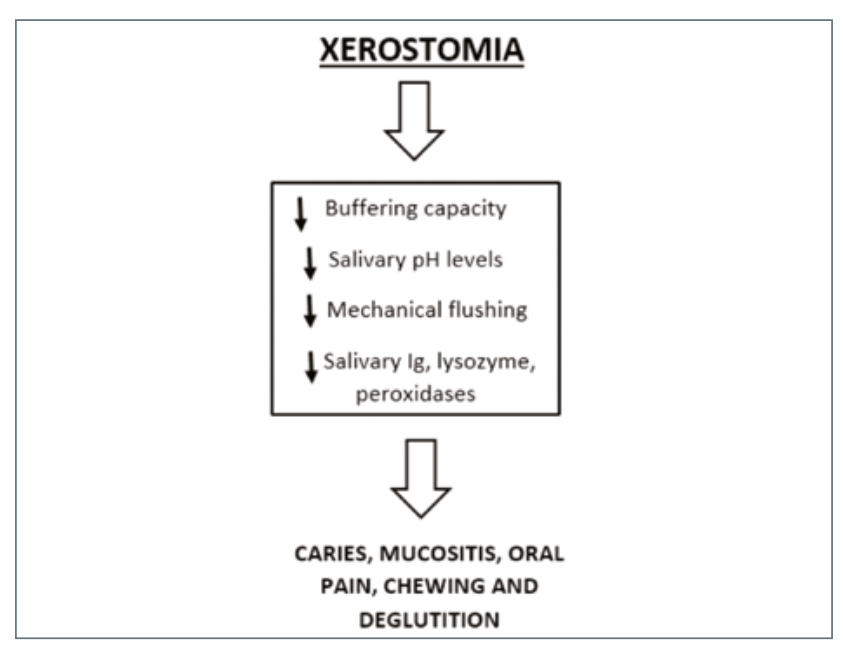

Figure 1. Xerostomia and clinical consequences. 
burning sensation, halitosis and intolerance to spicy foods, all of which can change a person's lifestyle ${ }^{8}$. In patients with removable dental prostheses, hyposalivation can often cause irritation which results in gingival mucosa damage. Saliva plays an important role in the perception of taste; actually, it is well known how a reduction of salivary flow can influence the ability to discriminate flavours, thus leading to the loss of appetite in the geriatric patient. All these symptoms can lead to changes in food and fluid selection which may compromise nutritional status. One of the main stimuli of saliva production is represented by the food direct activation on the chemoreceptors located at the level of the taste buds. Actually, the diet of the elderly affected by xerostomia consists mainly of soft or semi-liquid foods that do not require an abundant production of saliva for the formation of food bolus. Specifically, a reduced salivary output can disrupt food bolus formation and translocation. This alimentary behaviour causes the loss of taste bud stimulus which results in further reduction of saliva production. Geriatric patients also have a high choking hazard and an increased susceptibility to aspiration pneumonia, with consequent colonization of the lungs with gram-negative anaerobes from the gingival sulcus ${ }^{9}$. Extraoral signs include angular cheilitis when lips are dry, broken and frequently colonized with Candida species. After long periods of dehydration and general anaesthesia, older adults sometime develop acute parotitis. Swelling is quite recurrent and the glands may or may not be soft. Old patients sometimes complain about their tongue frequently becoming wrinkled, dry, and they have an increased susceptibility to developing microbial infections, particularly candidiasis ${ }^{10}$. Recurrent caries are particularly common in these patients due to retained natural teeth and previously restored dental surfaces. If there is a persistent salivary hypofunction, the salivary system is unable to maintain an adequate oral $\mathrm{pH}$ level, and this lack of $\mathrm{pH}$ regulation causes the microbial colonization of caries-associated microorganisms and enamel demineralization. Adults wearing dentures are more susceptible to traumatic lesions of desiccated and friable tissues due to the decrease of salivary proteins which provide oral lubrication. Eating and speech difficulties can compromise social relations and may cause some patients to avoid social interactions.

\section{MEDication}

Medication represents the most common cause of salivary gland disorders. Xerostomia is one of the most frequent side effects of drug therapy. Approximately $80 \%$ of commonly used drugs can cause salivary gland dysfunction ${ }^{11}$. As the need for medication increases with age, more than $75 \%$ of people aged 65 and older take at least one medication, therefore, drug-induced xerostomia is prevalent in the elderly ${ }^{12}$. The majority of drugs that cause salivary gland dysfunction have anticholinergic effects on the acinar cells through the inhibition of acetylcholine binding to muscarinic receptors. This blocks the onset of the cascade of physiological phases that, in the end, allows the water to move through the acinar cells into the ductal system, and, finally, into the mouth. A drug that disturbs ion transport pathways may also adversely affect the quality and quantity of salivary flow. Antihistamines, tricyclic antidepressants, sedatives and tranquillizers, antihypertensives (diuretics, alpha and beta blockers, calcium channel blockers, angiotensin converting enzyme inhibitors), cytotoxic agents, anti-Parkinsonian and antiseizure drugs may produce these salivary gland disorders ${ }^{13-17}$.

Among the most important inhibitors of salivation, antidepressant drugs have strong anticholinergic side effects. Amitriptyline and dothiepin cause more than a $50 \%$ reduction in stimulated parotid saliva ${ }^{18}$. Chemotherapy may also produce salivary gland disorders during and immediately after the treatment. These effects may be transitory or permanent ${ }^{19}$.

\section{HEAD AND NECK RT}

Head and neck RT has important and damaging side effects to the oral cavity. Patients often experience mouth dryness, dysgeusia, dysphagia, gingivitis, halitosis, chewing problems, mucositis and traumatic oral lesions ${ }^{20,21}$. Experiments show that the serous acini are the most radiosensitive cells due to cell death. More specifically, RT causes two types of damage, apoptosis at low doses and necrosis at high doses, due to the fact that the degenerative process proportionally increases with dose and time ${ }^{22}$. Gland parenchyma is characterized by low mitotic activity and cell death is due to deoxyribonucleic acid damage during and shortly after radiation. After a high radiation dose (60 Gy for head and neck cancer) changes become permanent and this causes atrophy of the salivary glands ${ }^{23}$. Thresholds of 23-25 Gy have been established. Above these levels, permanent salivary gland destruction occurs, and below them salivary gland recovery can occur after the completion of radiotherapy ${ }^{24}$.

\section{SJOGREN'S SYNDROME}

SS is a systemic autoimmune disease characterized by inflammation of epithelial tissues, especially exocrine glands. SS could be described as having primary and secondary forms. Primary SS involves lacrimal and salivary gland disorders associated with reduction of production of saliva and tears. Secondary SS manifests with other autoimmune diseases such as scleroderma, systemic lupus erythematosus, rheumatoid arthritis, 
and, polymyositis. Other epithelial components such as skin and the urogenital, respiratory, and gastrointestinal tracts are commonly involved. Thyroid disease is also common in SS patients. Incidence for primary SS ranges from 0.05 to $4.8 \%$ of the population ${ }^{25}$. SS may occur at any age, but diagnosis between 35 and 55 years of age is most common. Diagnosis may often be delayed for many years since the onset can be insidious ${ }^{26}$. The disease occurs in a greater number of females than in males, with a 9:1 ratio.

The pathogenesis of SS remains unknown ${ }^{27}$. An environmental agent (e.g., a virus) might trigger events in a susceptible host, resulting in the development of SS.

Since SS is far more frequent in women than in men, hormonal factors, including a relative lack of androgens, may influence pathogenesis ${ }^{28}$. A genetic component is also typical in SS. Prevalence of SS and autoantibodies (e.g., anti-Ro/SSA) may be higher in family members of people with SS than in the general population. Primary $S S$ in white patients is often related to the human leukocyte antigen (HLA)-DR3 and HLA-DQ2 alleles, whereas other alleles are more frequent in African-American and Japanese patients ${ }^{29}$. Mononuclear cell infiltrates in exocrine tissues and autoantibodies (particularly anti-Ro/SSA, anti-La/SSB and rheumatoid factor) are a characteristic of SS. The salivary and lacrimal infiltrates of T cells (CD4 helper cells), with fewer B cells, macrophages, and mast cells, could be observed in this disease ${ }^{30}$. The hallmarks of SS are ocular and oral symptoms and signs of dryness, a positive labial salivary gland biopsy, and the presence of autoantibodies (anti-Ro/SSA, anti-La/SSB). Insufficient tear production leads to inflammation and lacrimal gland damage. Major salivary gland swelling is frequent in SS due to ductal inflammation, salivary gland hypofunction, and acinar destruction. However, it is important to exclude malignancy in the presence of persistently and significantly enlarged salivary glands and neck lymph nodes. Needle aspiration (for cytological and flow cytometric analyses) associated with diagnostic imaging (e.g., computerized tomographic scans) are helpful in diagnosis. Laboratory tests in SS are frequently positive for anti-Ro/SSA or anti-La/SSB (50-90\%), rheumatoid factor $(90 \%)$ with the presence of hypergammaglobulinemia ${ }^{30}$. SS requires management of xerostomia, keratoconjunctivitis, and the syndrome's autoimmune and inflammatory manifestations. Replacement of damaged salivary gland tissue by artificial salivary glands ${ }^{31}$, immunomodulatory therapy ${ }^{32}$, and the possibilities for gene therapy are under active investigation. Nowadays, a multidisciplinary approach involving otolaryngologists, ophthalmologists, dentists, rheumatologists, and other medical experts is the best way to manage patients affected by SS.
Other autoimmune diseases that may cause xerostomia will be discussed more in detail in other chapters.

\section{MANAGEMENT OF XeROSTOMIA}

Frequent dental check-ups are often considered the first strategy in managing xerostomia due to the occurrence of complications ${ }^{32,33}$. Antimicrobial mouth rinses, low-sugar diet, and daily topical fluoride use help prevent caries in patients with insufficient salivary flow, furthermore remaining salivary gland secretions can be stimulated by candies, sugar-free chewing gum and mints. Artificial saliva and lubricants are used in addition to humidifiers for xerostomic symptom relief. Dysphagia is managed with oral moisturizers and lubricants, careful use of fluids during eating, and changes in diet. The Food and Drug Administration approved pilocarpine ${ }^{34}$ and cevimeline (two secretagogues) which are capable of increasing secretion and reducing xerostomic discomfort in patients with sufficient exocrine tissue.

Cevimeline should be preferred in therapy since it has a higher affinity for $\mathrm{M} 1$ and $\mathrm{M} 3$ muscarinic receptor subtypes. M2 and M4 receptors are located on cardiac and lung tissues so they can be used with minimum adverse effects on pulmonary and cardiac function, whereas Pilocarpine is a non-selective muscarinic agonist.

A frequent complication of dry mouth is oral candidiasis, which is commonly treated with topical antifungal agents ${ }^{35}$. Ointments, oral rinses, troches and pastilles are effective for most forms of oral candidiasis, and systemic antifungal therapy (e.g., ketoconazole, fluconazole) should be reserved for immunocompromised patients and refractory disease. Dental ill-fitting prosthesis may harbour fungal infections and this can be avoided by its immersion in solutions containing $1 \%$ sodium hypochlorite or benzoic acid, $0.12 \%$ chlorhexidine.

Substitution of xerostomia-associated drugs with similar types of medications with fewer xerostomic side effects is preferred. Tricyclic antidepressants have been reported to cause more mouth dryness than serotonin-specific reuptake inhibitors ${ }^{35}$. Milnacipran, an antidepressive drug and combined noradrenaline-serotonin reuptake inhibitor, provided improved outcomes with less dry mouth symptoms than clomipramine ${ }^{36}$. In treatment-resistant schizophrenia, olanzapine showed similar efficacy to chlorpromazine, yet it had 50\% less dry mouth side effects ${ }^{37}$. These examples and other substitutions can help improve drug-induced xerostomia.

Alternate pharmaceutical regimens may avoid xerostomia ${ }^{38}$. If anticholinergic drugs can be taken during the day, xerostomia can be reduced at night since salivary output is lowest at night. Assessment of drug side effects can help reduce the xerostomic potential of many pharmaceuticals used by older people.

Thereisstill noreal effectivetreatmentfor radiation-induced 
salivary gland dysfunction ${ }^{39}$. As noted, to achieve significant reductions in radiation exposure to glands, three-dimensional treatment planning and dose delivery techniques can be used in order to reduce xerostomia and to improve xerostomia-related quality of life ${ }^{40}$. Pilocarpine administration during and after the completion of radiotherapy can improve symptoms of xerostomia ${ }^{41}$. Amifostine, a cyto-radioprotectant, may provide cytoprotection against mucositis, nephrotoxicity, myelotoxicity, and xerostomia associated with various radiation and chemotherapy modalities ${ }^{42,43}$. These and other emerging strategies may further improve oral health-related quality of life of xerostomic elderly patients.

\section{PHARYNGO-LARYNGEAL TRACT}

Senescence is a process involving not only the whole human body but also the secretory system.

In order to understand senescence on the secretory glands of the pharyngo-laryngeal tract, the anatomy and functions of pharynx and larynx must be emphasized.

Although the pharynx mucosa has many caliciform mucosal cells and intraepithelial glands, most of the secretion of the pharyngo-laryngeal tract is produced by sub-mucous glands localized at the level of the larynx, as Gracco pointed out ${ }^{44}$.

These are exocrine glands set in a tubular and tubuloacinus structure. Tubular glands have a mucous secretion. Tubular-acinus glands are characterized by a serum-mucous ${ }^{45}$. Both of them are surrounded by myoepithelial cells. They develop at the level of the submucosa, with a specific distribution. In particular, a high concentration in sub-glottic region, in posterior glottic region (Figs. 2-3) and at the level of the supra-glottic region ${ }^{46}$ is observed.

In supraglottic region secretory glands are more widespread. In fact, they are not only found at the level of lamina propria and submucosa, but they even reach the area of the ligaments and muscles of this region. Serum secretion is prevalent in supra-glottis. A high glandular concentration is found at the level of false vocal folds and ventricles.

Glandular secretion has the function of lubricating the vocal folds, thus favouring phonation. By moisturizing the vocal cords, secretion guarantees the integrity of the cover. Other important functions are breath humidifying and ensuring the proper function of the mucociliary system. Secretion also plays a fundamental role in the mucosal immune defence mechanism, due to the production of immunoglobulins and other components ${ }^{47,48}$. In particular, IgA, lactoferrin and lysozyme that are the most important products for immune defence, are released in serum secretion by the tubuloacinar glands. Therefore, we can conclude that the supraglottic region is mainly involved in this function. Tomita et al. report how the glandular structures develop from the fourth month of gestation and that they are already complete at the fifth month of gestation ${ }^{47}$. During foetal life, at the level of the glandular epithelium, the production of a key protein for the mucosal immune system starts ${ }^{49-51}$. However, only in the post-natal period glands play a major role in the production of immunoglobulins ${ }^{52}$.

Glandular concentration and distribution in laryngeal tract increase during childhood and achieve their maximum level when larynx completes its anatomical development. Many studies show that the glandular concentration appears constant at the level of the sub-glottic and supra-glottic region up to the average age of 40 .

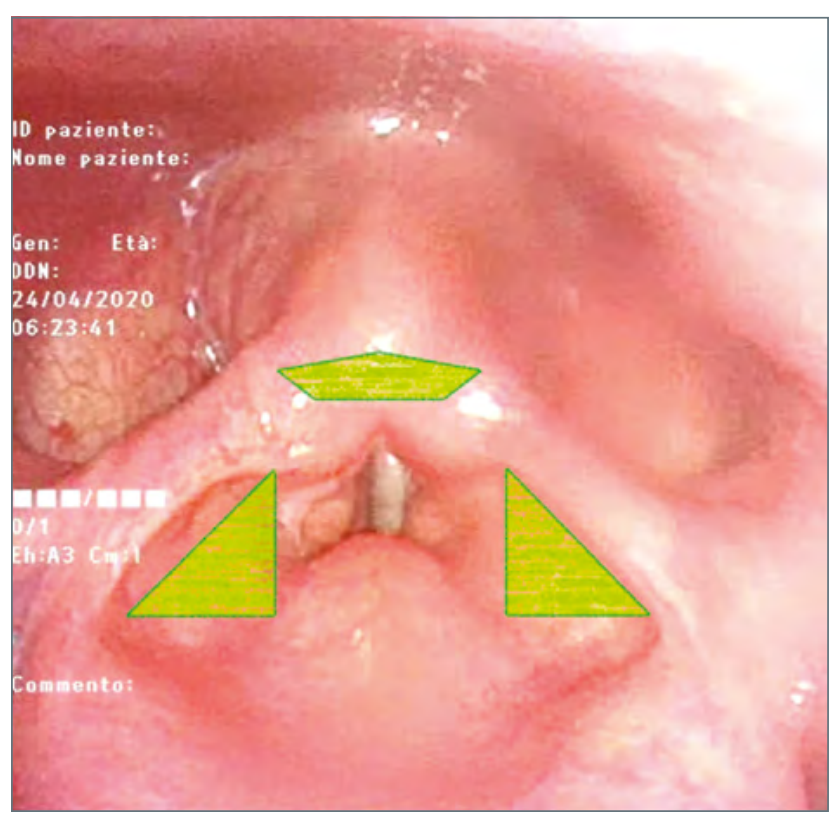

Figure 2. Salivary glands distribution in larinx.

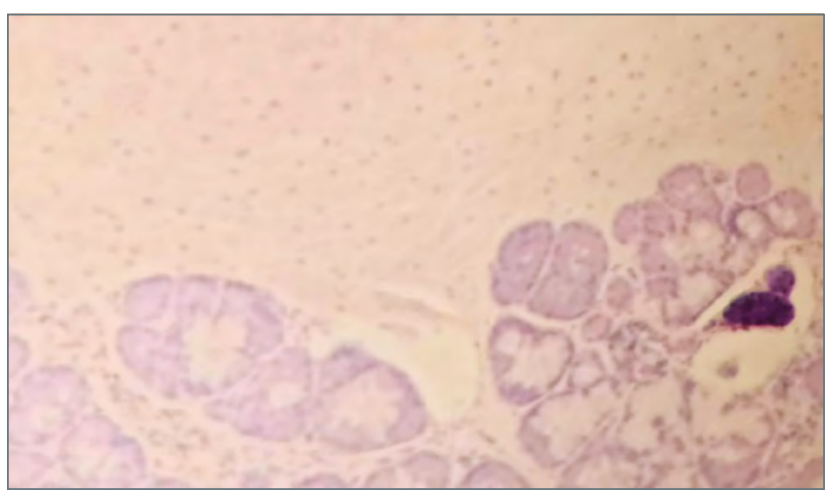

Figure 3. Salivary glands of larynx (posterior glottic region) [H\&E, original magnification 40x]. 
Subsequently, in subjects over 40 years old, a progressive reduction of the number of the glands in supraglottic region is described, while the concentration of glands at the subglottic level is steady ${ }^{53}$.

In the elderly the reduction of glandular tissue is also combined with tissue and intracellular changes. Intracellular changes are well documented by a study carried out by Sato et al. ${ }^{54}$. A decrease and atrophy of acini with replacement of the glandular cells with adipose tissue is detected. At the intracellular level, there is a reduction of rough endoplasmic reticulum (rER) and Golgi apparatus localized in basal region of the cytoplasm. In the apical zone of the cytoplasm, the secretory granules are reduced. Besides the total reduction of the number of granules, a change in quality can also be seen. In particular, electron-dense granules responsible of secretion of protein component, such as IgA ${ }^{50}$, lysozyme and lactoferrin (the main molecule of immune defence) are reduced in favour of electro-bright granules. This shows how, in the elderly, glandular secretion is not only generally reduced, but it assumes completely different characteristics from a qualitative viewpoint: it reduces the production of the protein components (electrondense granules) resulting in a reduced production of immune elements.

The reduction of the acini and, therefore, the decrease of serum component cause an imbalance in favour of the mucous component of secretion with consequent increase in terms of viscosity. This imbalance causes a negative impact on the muco-ciliary system. In addition, the decrease of protein elements such as immunoglobulins, lactoferrin and lysozyme, and the deficiency of the muco-ciliary system, expose the elderly to a greater vulnerability to external agents.

These qualitative and quantitative changes in glandular secretions play a key role in the functional senescence of the organ. At the laryngeal tract, in fact, secretion that is fundamental for the lubrication of mucosa also plays an important role for phonation.

Ichikawa ${ }^{55}$ performed photodynamic studies on a group of patients in order to evaluate the effects of the quantity and quality of mucus on the voice, concluding that viscosity of secretions has an impact on phonation. The Author assumes that morpho-functional changes in laryngeal salivary glands result in a quantitative reduction in secretions leading to a change in laryngeal viscosity and lubrication. This, along with the senile morphological variations of the vocal folds, is the basis of the vocal aging ${ }^{56}$. Moreover, the secretory modifications will lead the elderly to complain about dysphonia and pharyngo-laryngeal dryness in absence of organic laryngeal pathologies.

As it was already pointed out, another aspect playing an important role in the "organ aging" is the immunosenescence. It is known that laryngeal secretions contain immunoglobulins (mainly lgA, but also lgG and $\lg \mathrm{E}$ ) and antibacterial factors such as lactoferrin and lysozyme.

As we already mentioned, these components are secreted by the serous acicular glands and contain intracellular electron-dense granules. In the elderly, there is a reduction in the number of serum cells, which is particularly significant in the supra-glottic region. On one side, a reduction in electro-dense granules is observed and, on the other side, an increase in electro-lucent granules is also observed, which have different chemical and biological characteristics.

A qualitative and quantitative alteration of the granules leads to a reduction in the local immunity of the organ. Another fundamental defence mechanism in the upper airways is the muco-ciliary transport, which is regulated by three factors: eyelashes, mucus and their interaction. Increased viscosity of the mucus reduces the effectiveness of this system ${ }^{45}$. These two combined factors contribute to the reduction of organ defence mechanisms and an increase in the inflammatory and infectious phenomena of VADS.

In conclusion, we can say that secretory senescence in pharyngo-laryngeal tract is not only due to a decrease in number of exocrine glands, particularly in supra-glottic region, but also to an equally important alteration in quality. Reduction of serum protein secretion corresponds to an increase of mucus component which results in a decline of immune defence, higher viscosity and, consequently, a reduced efficacy of muco-ciliary system.

\section{References}

1 Dawes C, Pedersen AM, Villa A. The functions of human saliva: a review sponsored by the World Workshop on Oral Medicine VI. Arch Oral Biol 2015;60:863-74. https//doi. org/10.1016/j.archoralbio.2015.03.004

2 Miranda-Rius J, Brunet-Llobet L, Lahor-Soler E. Salivary secretory disorders, inducing drugs, and clinical management. Int J Med Sci 2015;12:811-24. https//doi. org/10.7150/ijms.12912

3 Melvin JE, Yule D, Shuttleworth T, et al. Regulation of fluid and electrolyte secretion in salivary gland acinar cells. Ann Rev Physiol 2005;67:445-69. https//doi.org/ 10.1146/annurev.physiol.67.041703.084745

4 Hall JE. Secretory functions of the alimentary tract. In Hall JE, Ed. Textbook of medical physiology, $12^{\text {th }}$ ed. Philadelphia: Saunders Elsevier 2011, pp. 773-6.

5 Catalán MA, Nakamoto T, Melvin JE. The salivary gland fluid secretion mechanism. J Med Invest 2009;56(Suppl):S192-6. https//doi.org/ 10.2152/jmi.56.192

6 Ship JA, Pillemer SR, Baum BJ. Xerostomia and the geriatric patient. J Am Geriatric Soc 2002;50:535-43. https//doi. org/ 10.1046/j.1532-5415.2002.50123.x. 
7 Gil-Montoya JA, Silvestre FJ, Barrios R, et al. Treatment of xerostomia and hyposalivation in the elderly: a systematic review. Med Oral Patol Oral Cir Bucal 2016;21:e355-66. https//doi.org/ 10.4317/medoral.20969

8 Atkinson JC, Wu A. Salivary gland dysfunction: causes, symptoms, treatment. J Am Dent Assoc 1994;125:409-16. https//doi.org/10.14219/jada.archive.1994.0059

9 Gibson G, Barrett E. The role of salivary function on oropharyngeal colonization. Spec Care Dent 1992;12:153-6. https//doi.org/ 10.1111/j.1754-4505.1992.tb00436.x

10 Terpenning M, Bretz W, Lopatin D, et al. Bacterial colonization of saliva and plaque in the elderly. Clin Infect Dis 1993;16(Suppl 4):S314-6. https/doi.org/ 10.1093/clinids/16.supplement_4.s314

11 Smith RG, Burtner AP. Oral side-effects of the most frequently prescribed drugs. Spec Care Dent 1994;14:96-102. https//doi.org/ 10.1111/j.1754-4505.1994.tb01112.x

12 Narhi TO. Prevalence of subjective feelings of dry mouth in the elderly. J Dent Res 1994;73:20-5. https//doi.org/ 10.1177/00220345940730010301

13 Nederfors T, Twetman S, Dahlof C. Effects of the thiazide diuretic bendroflumethiazide on salivary flow rate and composition. Scan J Dent Res 1989;97:520-7. https//doi. org/10.1111/j.1600-0722.1989.tb00926.x

14 Nederfors T, Dahlof C, Ericsson T, et al. Effects of the antihypertensive drug captopril on human salivary secretion rate and composition. Eur J Oral Sci 1995;103:351-4. https//doi.org/ 10.1111/j.1600-0722.1995.tb01856.x

15 Streckfus CF, Wu AJ, Ship JA, et al. Stimulated parotid gland salivary flow rates in normotensive, hypertensive, and hydrochlorothiazide-medicated African Americans. J Oral Path Med 1994;23:280-3. https//doi. org/10.1111/j.1600-0714.1994.tb00060.x

16 Nederfors T. Xerostomia: prevalence and pharmacotherapy. With special reference to beta-adrenoceptor antagonists. Swed Dent J(Suppl)1996;116:1-70.

17 Bergdahl M, Bergdahl J. Low unstimulated salivary flow and subjective oral dryness: Association with medication, anxiety, depression, and stress. J Dent Res 2000;79:1652-8. https//doi.org/10.1177/00220345000790090301

18 Hunter KD, Wilson WS. The effects of antidepressant drugs on salivary flow and content of sodium and potassium ions in human parotid saliva. Arch Oral Biol 1995;40:983-9. https//doi.org/10.1016/0003-9969(95)00079-5

19 Harrison T, Bigler L, Tucci M, et al. Salivary slgA concentrations and stimulated whole saliva flow rates among women undergoing chemotherapy for breast cancer: an exploratory study. Spec Care Dent 1998;18:109-12. https//doi. org/ 10.1111/j.1754-4505.1998.tb00914.x

20 Valdez $\mathrm{IH}$, Atkinson JC, Ship JA, et al. Major salivary gland function in patients with radiation-induced xerostomia: flow rates and sialochemistry. Int J Rad Oncol Biol Phys 1993;25:41-7. https//doi.org/ 10.1016/03603016(93)90143-j

21 Henson BS, Eisbruch A, D'Hondt E, et al. Two-year longitudinal study of parotid salivary flow rates in head and neck cancer patients receiving unilateral neck parotid-sparing radiotherapy treatment. Oral Oncol 1999;35:234-41. https//doi.org/10.1016/s1368-8375(98)00104-3

22 Stephens LC, King GK, Peters LJ, et al. Unique radiosensitivity of serous cells in rhesus monkey submandibular glands. Am J Pathol 1986;124:479-87.

23 Eisbruch A, Ten-Haken RK, Kim HM, et al. Dose, volume, and function relationships in parotid salivary glands following conformal and intensity modulated irradiation of head and neck cancer. Int J Rad Oncol Biol Phys 1999;45:577-87. https//doi.org/10.1016/s0360-3016(99)00247-3

24 Greenspan D. Oral complications of cancer therapies. Management of salivary dysfunction. $\mathrm{NCl}$ Monographs 1990;9:159-61.

25 Pillemer SR, Matteson EL, Jacobsson LT, et al. Incidence of physician-diagnosed primary Sjogren syndrome in residents of Olmsted County, Minnesota. Mayo Clin Proc 2001;76:593-9. https//doi.org/10.4065/76.6.593

26 Anaya JM, Talal N. Sjogren's syndrome comes of age. Semin Arthritis Rheumatol 1999;28:355-9. https//doi. org/10.1016/s0049-0172(99)80001-8

27 Fox Rl, Maruyama T. Pathogenesis and treatment of Sjogren's syndrome. Curr Opin Rheumatol 1997;9:393-9. https//doi.org/10.1097/00002281-199709000-00004

28 Sullivan DA. Sex hormones and Sjogren's syndrome. J Rheumatol 1997;24(Suppl 50):17-32.

29 Fox RI, Tornwall J, Maruyama T, et al. Evolving concepts of diagnosis, pathogenesis, and therapy of Sjogren's syndrome. Curr Opin Rheumatol 1998;10:446-56. https//doi. org/10.1097/00002281-199809000-00009

30 Anaya JM, Talal N. Sjogren's syndrome comes of age. Semin Arthritis Rheumatol 1999;28:355-9. https//doi. org/10.1016/s0049-0172(99)80001-8

31 Baum BJ, Wang S, Cukierman E, et al. Re-engineering the functions of a terminally differentiated epithelial cell in vivo. Ann NY Acad Sci 1999;875:294-300. https//doi. org/10.1111/j.1749-6632.1999.tb08512.x

32 Fox PC. Management of dry mouth. Dent Clin North Am 1997;41:863-76. https/doi.org/10.1254/jjp.82.281

33 Yamano S, Baum BJ. Prospects for gene-based immunopharmacology in salivary glands. Jap $\mathrm{J}$ Pharmacol 2000;82:281-6.

34 Vivino FB, Al-Hashimi I, Khan Z, et al. Pilocarpine tablets for the treatment of dry mouth and dry eye symptoms in patients with Sjogren syndrome: a randomized, placebocontrolled, fixed-dose, multicenter trial. P92-01 Study Group. Arch Intern Med 1999;159:174-81. https//doi. org/10.1001/archinte.159.2.174

35 Shay K, Truhlar MR, Renner RP. Oropharyngeal candidosis in the older patient. J Am Geriatr Soc 1997;45:863-70. https//doi.org/10.1111/j.1532-5415.1997.tb01517.x

36 Trindade E, Menon D, Topfer LA, et al. Adverse effects associated with selective serotonin reuptake inhibitors and tricyclic antidepressants: a metaanalysis. Can Med Assoc J 1998;159:1245-52.

37 Leinonen E, Lepola $U$, Koponen $H$, et al. Longterm efficacy and safety of milnacipran compared to clomipramine in patients with major depression. 
Acta Psychiatr Scand 1997;96:497-504. https//doi. org/10.1111/j.1600-0447.1997.tb09953.x

38 Conley RR, Tamminga CA, Bartko JJ, et al. Olanzapine compared with chlorpromazine in treatment-resistant schizophrenia. Am J Psychiatry 1998;155:914-20. https// doi.org/ 10.1176/ajp.155.7.914

39 Sreebny LM, Schwartz SS. A reference guide to drugs and dry mouth-2 ${ }^{\text {nd }}$ edition. Gerodontology 1997;14:33-47. https//doi.org/10.1111/j.1741-2358.1997.00033.x

40 Greenspan D. Oral complications of cancer therapies. Management of salivary dysfunction. $\mathrm{NCl}$ Monographs 1990;9:159-61.

41 Eisbruch A, Dawson LA, Kim HM, et al. Conformal and intensity modulated irradiation of head and neck cancer: the potential for improved target irradiation, salivary gland function, and quality of life. Acta Otorhinolaryngol Belg 1999;53:271-5.

42 Zimmerman RP, Mark RJ, Tran LM, et al. Concomitant pilocarpine during head and neck irradiation is associated with decreased posttreatment xerostomia. Int J Rad Oncol Biol Phys 1997;37:571-5. https//doi.org/10.1016/s03603016(96)00557-3

43 Capizzi RL, Oster W. Chemoprotective and radioprotective effects of amifostine: an update of clinical trials. Int J Hematol 2000;72:425-35.

44 Gracco C, Kahane JC. Age-related changes in the vestibular folds of the human larynx: a histomorphometric study. Journal of Voice 1989

45 Kutta H, Steven P, Kohla G, et al. The human false vocal folds - an analysis of antimicrobial defense mechanisms. Anat Embryol (Berl) 2002;205:315-23. https//doi. org/10.1007/s00429-002-0255-8

46 Ganly I, Patel SG, Coleman M, et al. Malignant minor salivary gland tumors of the larynx. Arch Otolaryngol Head Neck Surg 2006;132:767-70. https//doi.org/10.1001/ archotol.132.7.767
47 Nakashima T, Makishima K, Komiyama S, et al. Local immune system in the developing fetal larynx. Laryngoscope 1980;91:398-407. https//doi.org/10.1288/00005537198103000-00009

48 Nakashima T, Komiyama S, Makishima K, et al. Immunopathological study of the larynx. IgA distribution and secretory activity. Ann Otol Rhinol Laryngol 1980;89:35965. https//doi.org/10.1177/000348948008900412

49 Tomasi Jr TB, Jan EM, Solomon A, et al. Characteristics of an immune system common to certain external secretions. J Exp Med 1965;121:101-24. https//doi.org/10.1084/ jem.121.1.101

50 Brandtzaeg P. Mucosal and glandular distribution of immunoglobulin components. Differential localization of free and bound SC in secretory epithelial cells. J Immunol 1974;112:1553-9.

51 Mogi G, Watanabe N, Maeda S, et al. Laryngeal secretions. An immunological and immunohistological study. Acta Otolaryngol (Stockh) 1979;87:129-41. https//doi. org/10.3109/00016487909126397

52 Nakashima T, Umeno H, Yonekawa S, et al. Growth of laryngeal glands in I infants: a comparative study of the sub- and supraglottic larynx. J Laryngol Otol 2002;116:148. https//doi.org/10.1016/j.anl.2006.01.001

53 Tomita H, Nakashima T, Maeda A, et al. Age related changes in the distribution of laryngeal glands in the human adult larynx. Auris Nasus Larynx 2006;33:289-94. https//doi. org/10.1177/000348949810700612

54 Sato K, Hirano M. Age-related changes in the human laryngeal glands. Ann Otol Rhinol Laryngol 1998;107:525-9.

55 Ichikawa T. Basic studies on the lubrication of the larynx during phonation. Jibi to Rinsho 1982;28:38-52. https// doi.org/10.1258/0022215021910276

56 Davids T, Klein AM, Johns MM $3^{\text {rd }}$. Current dysphonia trends in patients over the age of 65: is vocal atrophy becoming more prevalent? Laryngoscope 2012;122:332-5. https//doi.org/10.1002/lary.22397 